East African Medical Journal Vol. 81 No. 3 March 2004

MORPHOLOGICAL STUDY OF THE UNCOMMON RECTUS STERNI MUSCLE IN GERMAN CADAVERS

M. A. Motabagani, PhD., A. Sonalla, PhD., E. Abdel-Meguid PhD., and M. A. Bakheit, PhD., Department of Anatomy, College of Medicine, King Faisal University, P.O Box 2114, Dammam, 31451, Saudi Arabia

Request for reprints to: Dr. M. A Motabagani, Department of Anatomy, College of Medicine, King Faisal University, P.O. Box 2114, Dammam, 31451, Saudi Arabia

\title{
MORPHOLOGICAL STUDY OF THE UNCOMMON RECTUS STERNI MUSCLE IN GERMAN CADAVERS
}

\author{
M. A. MOTABAGANI, A. SONALLA, E. ABDEL-MEGUID \\ and M. A. BAKHEIT
}

\begin{abstract}
Objective: The present investigation has been designed to study the incidence of the rectus stern muscle in German human cadavers dissected in the Kingdom of Saudi Arabia, trying to find a postulation for the development of such muscle when present. Design: Gross dissection of 130 cadavers, of both sexes, was performed throughout a period of 10 years.

Setting: Department of Anatomy College of Medicine, King Faisal University, Dammam, Saudi Arabia.

Intervention: Investigation of the origin and insertion of the rectus sterni and measurements of its length and width.

Results: Two adult cadavers, one of each sex, had shown well-developed bilateral rectus stern muscles. All muscles identified were parasternal in position, being superficial to the medial portion of the pectoralis major muscle. Minor morphological differences were observed among the four muscle masses concerning their length, breadth, origin and insertion.

Conclusion: The current study has determined the incidence of the rectus sterni muscle, in German cadavers to be $1.54 \%$ per bodies examined compared to $4 \%$ in cadavers from Saudis. Such a frequency is compared to that reported in different geographic populations. The rectus sterni muscle is innervated by the anterior cutaneous branches of the intercostal nerves. The description of the rectus sterni muscle and its incidence determined in the present study, might be of a great help for clinicians radiographing or tackling the pectoral region.
\end{abstract}

\section{INTRODUCTION}

The rectus sterni muscle has been identified as an infrequent mass of striated musculature in front of the human chest(1). The muscle, when present, received other names as the sternalis, the episternalis, the rectus thoracis and the superficial rectus abdominis(2). The prevalence of the rectus sterni muscle has been reported to vary widely among different nationalities, from $1 \%$ in Taiwanese(3) to $23.5 \%$ in Chinese(4). Certain morphological and mammographic studies, determined the incidence of this muscle to be $4 \%$ in the Kingdom of Saudi Arabia(5).

The laterality of the rectus sterni muscle has been also investigated by many authors. It is twice unilateral than bilateral(6). The rectus sterni muscle has been classified into unilateral and bilateral with four subtypes for each type group(7). When identified, the muscle is described as thin, flat and long arising from the anterior chest wall, below the clavicle, running parallel to the sternum but superficial to the medial part of the rectus abdominis muscle, and finally gaining insertion into the costal cartilages, the lower end of the sternum or the external oblique aponeurosis of the abdomen $(1,8)$.

It has been phylogenetically suggested that there is a longitudinal ventral paramedian muscle sheet that disappears leaving the hyoid muscles in the neck and the rectus abdominis muscle as representatives. The rectus sterni muscle is a remnant of this longitudinal muscle in the anterior thoracic wall although it has been always demonstrated to be superficial to but not continuous with the rectus abdominis muscle(9).

In certain mammographic studies the rectus sterni muscle appeared as an irregular focal density along the medial aspects of the mammograms, giving a false perception of a breast mass $(10,11)$. The aim of the current study was to determine the incidence of the German rectus sterni muscle dissected in Saudi Arabia and to describe, in details, the morphology of this muscle when identified. The results of this study might highlight the reported postulations concerning its development. Furthermore, the exact location and extension of this muscle would be of a great concern to clinicians when interpreting mammograms. 


\section{MATERIALS AND METHODS}

One hundred and thirty formalin fixed adult German cadavers, of both sexes over a period of 10 years, were utilised in this study. They were used for teaching the medical students in the Anatomy Laboratory, College of Medicine, King Faisal University, Dammam, Saudi Arabia. Fine dissection of the pectoral region was carefully performed. Any muscular variant in the paramedian region of the anterior chest wall was looked for and followed for its origin, insertion, nerve supply and morphological features. The length and breadth of the muscle, along its whole extension, were determined using a thread that was then applied to a ruler.

\section{RESULTS}

Two cadavers, one of each sex, out of 130 formalin-fixed German human cadavers over a 10 year period, were observed to exhibit bilateral rectus sterni muscles.

Two well-demarcated masses of an asymmetrical bilateral rectus sterni muscle were observed in a male adult cadaver. Each muscle mass was located on the anterior chest wall, in a paramedian position, just deep to the skin and superficial fascia of the pectoral region, but superficial to the pectoral fascia and the pectoralis major muscle. The left muscle measured $11 \mathrm{~cm}$ long and $2 \mathrm{~cm}$ broad. It arose from the left part of the front of the manubrium sterni and from the sternal tendon of the left sternocleidomastoid muscle. This left rectus sterni muscle descended vertically to gain insertion into the left 5th and 6th costal cartilages and the aponeurosis of the left external oblique muscle of the abdomen taking share in the formation of the anterior wall of the left rectus sheath (Figures 1-3).

\section{Figure 1}

Morphological types of the rectus sterni muscle as described by Jelev et al. (2001)
Figure 2

A photograph (A) and a schematic diagram (B) of an asymmetrical bilateral rectus sterni muscles (RS) of specimen I, showing their location and extension. The right muscle is larger than the left one. Note the sternocleidomastoid (SM) and pectoralis major (PM) muscles

Figure 3

A photograph (A) and a schematic diagram (B) of the magnified proximal parts of the rectus sterni muscles $(R S)$ shown in figure 2, revealing the origin of each muscle from the corresponding sternal tendon of the sternocleidomastoid muscle (SM). The rectus sterni muscles are located superficially to the pectoralis major muscles $(\mathrm{PH})$ 
The right rectus sterni muscle, demonstrated in the same cadaver, was larger than the left one. It measured $12 \mathrm{~cm}$ long and $2.5 \mathrm{~cm}$ at its broadest part. It had an origin similar to that of its left counterpart but it slightly inclined to the right near its lower end to gain insertion into the right 5 th, 6 th and 7 th costal cartilages and the right external oblique aponeurosis.

As regards the arterial supply and innervation of the left rectus sterni muscle, it was found that the perforating branches of the left internal thoracic artery and the anterior cutaneous branches of the left 2nd, 3rd and 4th intercostal nerves were noticed to it. The left pectoral nerves were observed to give no branches to the left rectus sterni muscle. The superficial and deep relations of the right rectus sterni as well as its arterial supply were exactly similar to those of the left muscle. The right muscle was innervated by the anterior cutaneous branches of the right 3 rd and 4 th intercostal nerves.

The pectoralis major muscle, on each side, was morphologically normal without presenting any anomaly or variation.

\section{Figure 4}

\footnotetext{
A photograph (A) and a schematic diagram (B) of the asymmetrical bilateral rectus sterni muscles (RS) of specimen II, showing the right muscle is larger than the left one. The left rectus sterni muscle occupies an infraclavicular position without any apparent continuity with the left sternocleidomastoid muscle (SM). Note the pectoralis major muscles (PM)
}

\section{Figure 5}

A photograph (A) and a schematic diagram (B) of the magnified proximal parts of the rectus sterni muscles $(R S)$ shown in figure 4, revealing their asymmetry in origin and size. The sternocleidomastoid (SM) and pectoralis major (PM) muscles are also shown
Another asymmetrical bilateral rectus sterni muscle was demonstrated in a female adult cadaver. The left rectus sterni muscle, in this specimen, was relatively smaller measuring $8 \mathrm{~cm}$ long and $1.5 \mathrm{~cm}$ broad. It extended from the left margin of the sternal angle down to the left 5th costochondral junction.

The right muscle mass had a proximal tendinous part $(7 \mathrm{~cm})$ and a distal fleshy part $(5 \mathrm{~cm})$ with a maximal breadth of $2.5 \mathrm{~cm}$. It also arose from the sternal tendon of the sternocleidomastoid muscle, and gained insertion into the right 5th and 6th costal cartilage and the right external oblique aponeurosis.

As regards to the arterial supply and innervation of the left rectus sterni, it was found that this muscle was supplied by the perforating branches of the left internal thoracic artery and innervated by the left 3rd and 4th intercostal nerves. The right rectus sterni received supply from perforating branches of the right internal thoracic artery and innervation from the anterior cutaneous branches of the right 4 th and 5 th intercostal nerves.

Both muscles, in specimen II, assumed the same vertical position, relation and location as the rectus sterni muscles of specimen I (Figures 4-5). 


\section{DISCUSSION}

The rectus sterni muscle has been investigated by many authors in different populations. It is assumed that this muscle is developed from one or more of the neighbouring muscles such as the pectoralis major muscle $(12,13)$ the sternocleidomastoid muscle(14), or the rectus abdominis muscle(15). Furthermore, the previous authors suggested that the rectus sterni muscle has the same innervation as the muscle of origin. Some authors agreed that the rectus sterni muscle is innervated by the pectoral nerves while other investigator demonstrated its nerve supply from the intercostal nerves and sometimes the muscle had dual innervation indicating dual origins(13).

The rectus sterni muscle has been demonstrated, in the present study, to be supplied by the anterior cutaneous branches of the intercostal nerves and the perforating branches of the internal thoracic artery. Such findings are not consistent with the old reports which suggested that the rectus sterni muscle is supplied by the pectoral nerves(16-18) but the findings of the present study run in accordance with the recent reports of other workers $(3,7,19)$.

The present study has demonstrated two specimens with bilateral rectus sterni muscles innervated solely by the intercostal nerves. Communicating twigs were not observed between the pectoral and intercostal nerves, in the specimens investigated. The present work, therefore, supports the opinion that the rectus sterni muscle is derived from the rectus abdominis muscle as the two muscles have the same innervation. The results of this study also run in accordance to the proposal that a ventral longitudinal paramedian muscular sheet is represented, in human being, by the hyoid muscles in the neck, the rectus abdominis muscle in the abdomen, and occasionally the rectus sterni muscle in the thorax $(9,19)$.

Based on a previous classification(7), the current first specimen represents subtype while the second specimen represents subtype of the bilateral group of the rectus sterni muscles.

The present work determined the incidence of the rectus sterni muscle is $1.54 \%$ per German cadavers dissected in Saudi Arabia. The frequency however, has been reported to vary widely from $1 \%$ in Taiwanese $(3,16)$ to $23.5 \%$ in Chinese(4). This muscle has been previously reported to have an incidence of $4 \%$ in the cadaveric specimens in Saudi Arabia(5).

The present investigation provides a detailed morphology of the rectus sterni muscle. The incidence of such uncommon muscle is determined to be $1.54 \%$ amongst German cadavers compared to $4 \%$ in Saudis.
Moreover, this work suggests that the rectus sterni muscle is derived from the rectus abdominis muscle as the two muscle masses are supplied by the segmental intercostal nerves. The results of the present study might be of great concern to radiologists and surgeons dealing with the pectoral region.

\section{REFERENCES}

1. Bailey, P.M. and Tzarnas, C.D. The sternalis muscle: a normal finding encountered during breast surgery. Plast. Reconstl. Surg. 1991; 103:1189-1190.

2. Kida, M.Y., Izumi, A. and Tanaka, S. Sternalis muscle: Topics for debate. Clin. Anat. 2000; 13:138-140.

3. Jeng, H. and Su, S.J. The sternalis muscle: An uncommon anatomical variant among Taiwanese. J. Anat. 1998; 193:277-286.

4. Fukuyama, U. Der musculus sternalio bei den Norchinesen. Okjimas Dolica Anatomica Jpn. 1940; 19:69-92.

5. Saeed, M., Murshid, K., Rufai, A., El-Sayed, S. and Sadig, M. Sternalis: An anatomic variant of chest wall musculature. Saudi Med. J. 2002; 23:1214-1221.

6. Bergman, R.A., Thompson, S.A., Afili, A.K. and Saadeh, F.A. Compendium of human anatomic variation. Urban and Schwarzenberg. Munich, 1988.

7. Jelev, L., Georgie, G. and Suchw, L. The sternalis muscle in the Bulgarian Population. Classification of sternalis. J. Anat. 2001; 1991:359-363.

8. Williams, S.P.L., Bannister, I.H., Berry, M.M., Dyson, M., Dussek, J.E. and Ferguson, M.W. Gray's Anatomy - 38th Edition, Churchill Livingstone Edinburgh, Scotland, 1995.

9. Sadler, T.W., Langman's Medical Emblyology - 7th Edition, Williams. Baltimore, 1991.

10. Britton, C.A., Baratz, A.B. and Harris, K.M. Carcinoma mimicked by the sternal insertion of the pectoral muscle. Amer. J. Roentgenol. 1989; 153:955-965.

11. Britton, C.A. Subpectoral mass mimicking a malignant breast mass on mammography. Amer. J. Roentgenol. 1992; 159:221-227.

12. Clement, C.D. Muscle and fascia; In Gray's Anatomy - 36th Edition, Lea and Febiger. Philadelphia. 1985.

13. Kida, M.Y. and Kudoh, H. Innervation of the sternalis muscle accompanied by congenital partial absence of the pectoralis major muscle. Okajimas Folia Anat. Jpn. 1991; 67:449-455.

14. Bless, G. A peculliar type of sternalis. Acta Morphol. Neerl. Scand. 1968; 7:69-72.

15. Larsen, W.J. Human Embryoloy, Churchill Livingstone. Edinburgh, Scotland. 1997.

16. Shen, C.L., Chell, C.H. and Lee, S.H. A Taiwanese with a pair of sternalis muscle. Kaibogakin Zasshi. 1992; 67:652-654.

17. Ura, R. A personal interpretation of the origin and nature of sternalis muscle. Acta Anat. Jpn. 1938; 7:64-65.

18. Morita, M. Observations of the muscles sternalis and muscli pectoralis in mammals and a morphological interpretation of the essence of musculus sternalis. Acta Anat. Jpn. 1994; 22:357-366.

19. O'Nall, M. and Fallen-Curran, J. Case report: Bilateral sternalis muscle with a bilateral pectoralis major anomaly. J. Anat. 1998; 193:289-292. 